\title{
RECENT ADVANCES IN SMAP RFI PROCESSING
}

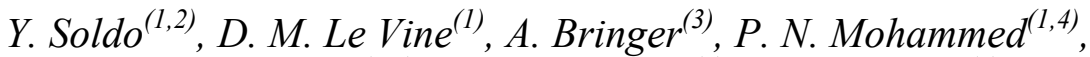 \\ P. de Matthaeis ${ }^{(1,2)}$, J. R. Piepmeier ${ }^{(1)}$, J. T. Johnson ${ }^{(3)}$ \\ (1) NASA Goddard Space Flight Center, Greenbelt, MD, USA \\ (2) Universities Space Research Association, Columbia, MD, USA \\ (3) Ohio State University, Columbus, OH, USA \\ (4) Morgan State University, Baltimore, MD, USA
}

\begin{abstract}
The measurements made by the Soil Moisture Active/Passive (SMAP) mission are affected by the presence of Radio Frequency Interference (RFI) in the protected 1400-1427 MHz band. In SMAP data processing, the main protection against RFI is a sophisticated RFI detection algorithm which flags sub-samples in time and frequency that are contaminated by RFI and removes them before estimating the brightness temperature. This contribution presents two additional approaches that have been developed to address the RFI concern in SMAP. The first consists in locating sources of RFI; once located, it becomes possible to report RFI sources to spectrum management authorities, which can lead to less RFI being experienced by SMAP in the future. The second is a new RFI detection method that is based on detecting outliers in the spatial distribution of measured antenna temperatures.
\end{abstract}

Index Terms - Soil Moisture Active/Passive (SMAP), Radio Frequency Interference (RFI)

\section{INTRODUCTION}

The Soil Moisture Active/Passive (SMAP) mission was launched by NASA on January $31^{\text {st }}, 2015$. Its main goals are to measure soil moisture and to determine the frozen or thawed state of the surface [1]. In order to do this, SMAP was equipped with a radiometer operating in the 1400-1427 $\mathrm{MHz}$ band, which is allocated to passive uses only. However, the radiometers onboard SMOS and Aquarius, that operate in the same frequency band had already reported significant contamination from Radio-Frequency Interference (RFI) sources [2], [3]. Due to SMAP's later launch, it was possible to equip SMAP with hardware and software better suited for RFI detection. This resulted in a sophisticated detection algorithm [4] that is included in SMAP's data processing.

This contribution presents how the RFI detection algorithm has been used to locate and report the locations of RFI sources, and how the occurrence of RFI signals missed by this algorithm can be reduced by introducing a new RFI flag.

\section{RFI DETECTION ALGORITHM}

SMAP has two types of data streams. One is referred to as "full-band" and corresponds to the entire bandwidth of the SMAP filter, $24 \mathrm{MHz}$, and to an integration time of about $300 \mu \mathrm{s}$. The other is called "sub-band" and consists of 16 frequency channels each corresponding to $1.5 \mathrm{MHz}$ and an integration time of about $1.2 \mathrm{~ms}$ [4], [5]. For each data stream, SMAP takes measurements of all four modified Stokes parameters (V-pol, H-pol, $3^{\text {rd }}$ and $4^{\text {th }}$ Stokes parameters).

The RFI detection algorithm uses both data streams and all four modified Stokes parameters. The criteria used to detect RFI are the following:

- Time-domain outlier detection (V- and H-pol; both full-band and sub-band data);

- Kurtosis detection (V- and H-pol; both full-band and sub-band data);

- Cross-frequency outlier detection (V- and H-pol; sub-band data only);

- Polarization detection $\left(3^{\text {rd }}\right.$ and $4^{\text {th }}$ Stokes parameters; both full-band and sub-band data).

The outputs of this algorithm are all the RFI flags raised by the detection criteria above and the filtered antenna temperature (Ta_filtered), i.e., the antenna temperature averaged at every footprint after all the flagged subsamples have been removed.

\section{LOCALIZATION AND REPORTING OF RFI SOURCES}

Using the output of the RFI detection algorithm, it is possible to estimate the location of the sources of RFI. The SMAP team can then report the presence and location of 
RFI sources to the relevant spectrum management authorities.

\subsection{Localization}

The location of RFI sources is obtained with the help of two independent algorithms, which increases the reliability of the reported RFI locations.

The first algorithm, developed at the Goddard Space Flight Center, provides estimates for the actual location of an emitter on the ground. This algorithm uses the reported Ta_filtered to remove the natural background, allowing it then to focus on measurements affected by RFI. Once the background is removed, a clustering algorithm is applied to determine the number and approximate location of the RFI sources. A more accurate location is then determined by computing the centroid of the distribution of Ta-Ta_filtered within the clusters [6].

The second algorithm, developed at The Ohio State University provides the latitude and longitude of rectangular "boxes" corresponding to high RFI contamination. To define these boxes, the global map is divided into $0.25 \times 0.25$ degree cells, and cells are flagged if the difference between Ta and Ta_filtered is higher than $10 \mathrm{~K}$ in at least $25 \%$ of measurements. When multiple flagged cells are connected on four sides, a "box" is formed, defined as the minimum bounding rectangle including all the connected flagged cells.

Only the RFI locations provided by the first algorithm that are also inside the boxes provided by the second algorithm are considered for reporting.

\subsection{Reporting}

To date, the SMAP mission has reported RFI source locations in China and Japan to the applicable authorities. The report was performed in the context of a joint effort between NASA and ESA (European Space Agency) in December 2016 to report RFI sources in the 1400-1427 $\mathrm{MHz}$ band. In Figure 1 are plotted monthly averages of RFI percentages over China from November 2016 to November 2017 (RFI percent is defined as the percent of samples flagged as RFI by the algorithm in section 2). The figure shows a decreasing trend beginning in Dececmber 2016, although an increasing trend is present in the last few months.

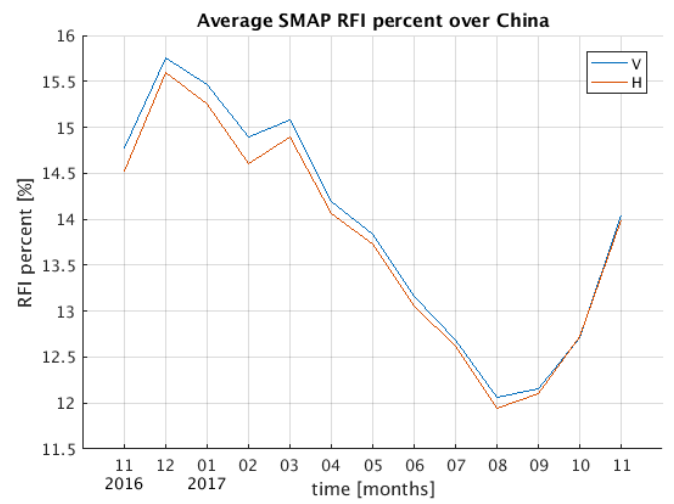

Figure 1: Time series of the SMAP RFI percent over China.

\section{AN ADDITIONAL APPROACH TO DETECT RFI CONTAMINATION}

Despite SMAP's extensive RFI processing, some RFI apparently remains undetected. In such cases, Ta_filtered is still affected by RFI so that its value remains increased with respect to the geophysical background by the contribution of the man-made signal. An additional RFI detection strategy has been devised to help address this issue. The new approach seeks to detect and flag outliers in the spatial distribution of Ta_filtered.

The first step of the new strategy is to use the Ta_filtered of individual orbits to draw contour lines corresponding to constant levels of Ta filtered. The contour lines are marked as RFI if three criteria are met: 1) the contour line corresponds to a local maximum; 2) the Ta associated with footprints inside the contour line has an average RFI percent higher than $10 \%$ (i.e., more than $10 \%$ of the measurments in the 8 time by 16 frequency subsample "spectrogram" of a given footprint are flagged); and 3) at least two contour lines are close (i.e., the ratio between their areas is lower than 1.5). Finally, wherever a contour line is marked as RFI, all footprints with centers within a $50-\mathrm{km}$ radius from the center of the contour line are flagged as RFI corrupted.

Figure 2 shows the spatial distribution of Ta_filtered for one half-orbit on July $5^{\text {th }}, 2017$, over a region heavily contaminated by RFI. In both panels, the samples flagged by the RFI detection algorithm or by any of the quality flags available in the SMAP L1B_TB products have been removed. In the bottom panel, the footprints flagged by this new approach have been also removed.

Notice that the two warm spots at about $40^{\circ}$ North are not detected as RFI by the current implementation, but are flagged with the new approach. The algorithm takes advantage of the spatially isolated nature of the warm spots to help identify RFI. 

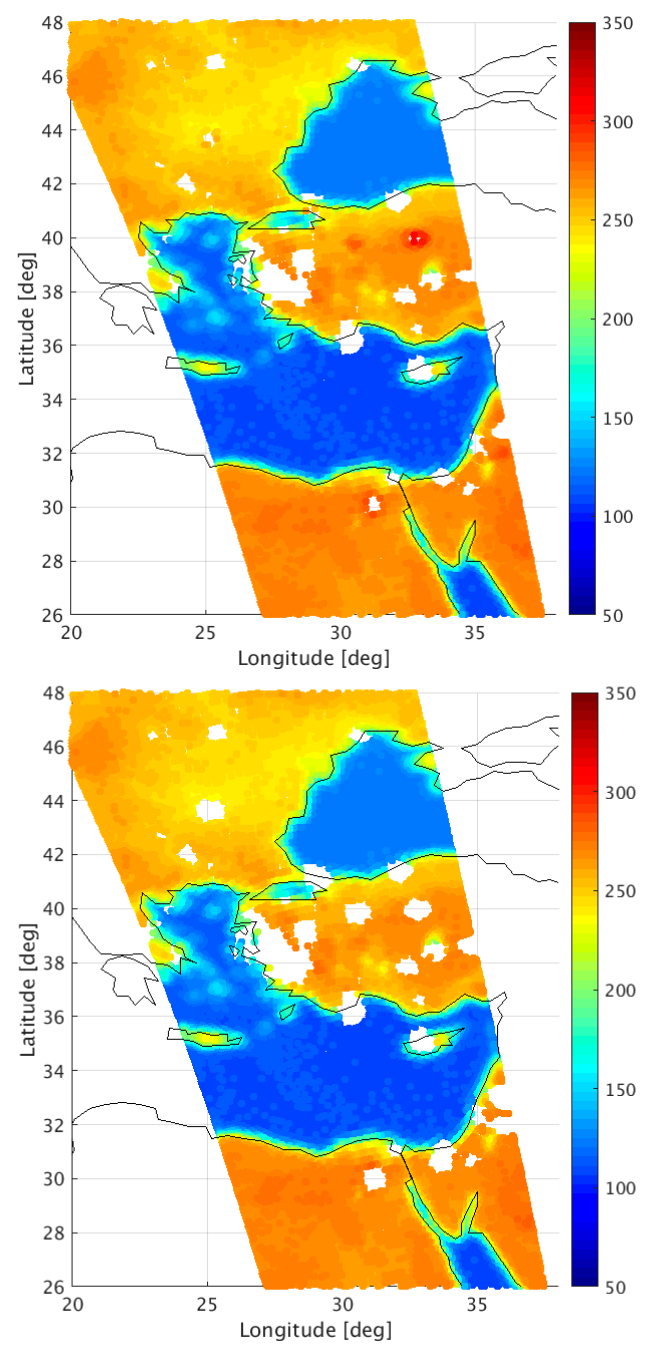

Figure 2: Map of the antenna temperature (V-pol) measured by SMAP during a pass on July $5^{\text {th }}, 2017$. In both panels, the samples flagged by the RFI detection algorithm or by the quality flags have been removed. In the bottom panel, the footprints flagged by the new RFI detection approach have been removed as well.

\section{REFERENCES}

[1] D. Entekhabi, E. G. Njoku, P. E. O'Neill, K. H. Kellogg, W. T. Crow, W. N. Edelstein, J. K. Entin et al., "The soil moisture active passive (SMAP) mission," Proceedings of the IEEE 98, no. 5, pp. 704-716, 2010.

[2] E. Daganzo-Eusebio, R. Oliva, Y. H. Kerr, S. Nieto, P. Richaume, and S. M. Mecklenburg, "SMOS radiometer in the 1400-1427-MHz passive band: Impact of the RFI environment and approach to its mitigation and cancellation," IEEE Transactions on Geoscience and Remote Sensing 51, no. 10, pp. 4999-5007, 2013.

[3]

D. M. Le Vine, P. de Matthaeis, C. S. Ruf, and D. D.

Chen, "Aquarius RFI detection and mitigation algorithm:

Assessment and examples," IEEE Transactions on Geoscience and Remote Sensing 52, no. 8, pp. 4574-4584, 2014.
[4] J. R. Piepmeier, J. T. Johnson, P. N. Mohammed, D. Bradley, C. Ruf, M. Aksoy, R. Garcia, D. Hudson, L. Miles, and M. Wong, "Radio-frequency interference mitigation for the soil moisture active passive microwave radiometer," IEEE

Transactions on Geoscience and Remote Sensing 52, no. 1, pp. 761-775, 2014

[5] SMAP Project: Algorithm Theoretical Basis Document, SMAP L1B Radiometer Brightness Temperature Data Product: L1B_TB (Rev. B), section 3, 2016.

[6] Y. Soldo, D. Le Vine, A. Bringer, P. de Matthaeis, R. Oliva, J. T. Johnson and J. Piepmeier, "Location of Radio Frequency Interference sources using the SMAP L-band radiometer," IEEE Transactions on Geoscience and Remote Sensing (submitted). 